\title{
Polynomial cases of the tarification problem
}

\author{
Citation for published version (APA):
}

van Hoesel, C. P. M., van der Kraaij, A. F., Mannino, C., Oriolo, G., \& Bouhtou, M. (2003). Polynomial cases of the tarification problem. METEOR, Maastricht University School of Business and Economics. METEOR Research Memorandum No. 053 https://doi.org/10.26481/umamet.2003053

Document status and date:

Published: 01/01/2003

DOI:

10.26481/umamet.2003053

Document Version:

Publisher's PDF, also known as Version of record

\section{Please check the document version of this publication:}

- A submitted manuscript is the version of the article upon submission and before peer-review. There can be important differences between the submitted version and the official published version of record.

People interested in the research are advised to contact the author for the final version of the publication, or visit the DOI to the publisher's website.

- The final author version and the galley proof are versions of the publication after peer review.

- The final published version features the final layout of the paper including the volume, issue and page numbers.

Link to publication

\footnotetext{
General rights rights.

- You may freely distribute the URL identifying the publication in the public portal. please follow below link for the End User Agreement:

www.umlib.nl/taverne-license

Take down policy

If you believe that this document breaches copyright please contact us at:

repository@maastrichtuniversity.nl

providing details and we will investigate your claim.
}

Copyright and moral rights for the publications made accessible in the public portal are retained by the authors and/or other copyright owners and it is a condition of accessing publications that users recognise and abide by the legal requirements associated with these

- Users may download and print one copy of any publication from the public portal for the purpose of private study or research.

- You may not further distribute the material or use it for any profit-making activity or commercial gain

If the publication is distributed under the terms of Article $25 \mathrm{fa}$ of the Dutch Copyright Act, indicated by the "Taverne" license above, 


\title{
Polynomial cases of the tarification problem
}

\author{
Stan van Hoesel * $\quad$ Anton F. van der Kraaij ${ }^{\dagger} \quad$ Carlo Mannino ${ }^{\ddagger}$ \\ Gianpaolo Oriolo § Mustapha Bouhtou ^
}

December 18, 2003

\begin{abstract}
We consider the problem of determining a set of optimal tariffs for an agent in a network, who owns a subset of the arcs of the network, and who wishes to maximize his revenues on this subset from a set of clients that make use of the network.

The general variant of this problem is $\mathcal{N} \mathcal{P}$-hard, already with a single client. This paper introduces several new polynomially solvable special cases. An important case is the following. For multiple clients, if the number of tariff arcs is bounded from above, we can solve the problem by a polynomial number of linear programs (each of which is of polynomial size). Furthermore, we show that the parametric tarification problem and the single arc fixed charge tarification problem can be solved in polynomial time.

Subject Classification: Networks, Combinatorial Optimization, Bilevel Programs.
\end{abstract}

\section{Introduction}

The tarification problem in a network involves two non-cooperative groups, tariff setting agents and tariff following clients. Each arc in the network is owned by at most one agent. Being the owner of an arc an agent can set the price for renting capacity on the arc freely, in order to maximize his revenues. The clients wish to route a certain demand for capacity on a path connecting two vertices (a commodity). A selected path can involve connections belonging to different agents. Clearly, each client will select a path with minimum cost to satisfy the demand for his commodity.

We restrict the problem to a single agent who knows the tariffs of his competitors and intends to charge revenue-maximizing tariffs on the subset of the network arcs owned by himself. This agent is generally referred to as the leader, while the clients are referred to as followers. The arcs that are not owned by the leader belong to the other agents in the network.

An elegant and natural formulation of this problem is a bilevel program introduced in Labbé et al. [4], where at the upper level the leader strives to maximize his revenue, while at the lower level the clients seek to minimize the cost of routing their demands. In this formulation, the objective functions of the leader and the clients are bilinear. For the tarification problem with linear pricing of the tariff arcs, Labbé et al. [4] showed that the single agent problem is $\mathcal{N} \mathcal{P}$-hard, even for one commodity, but with lower bounds on the tariffs. Recently, Roch et al. [5] have refined this result and shown that the problem is $\mathcal{N} \mathcal{P}$-hard for one commodity and nonnegative tariffs. Roch et al. [5], propose an approximation algorithm for the single-commodity case with linear pricing strategies and a performance guarantee of $\frac{1}{2} \log |T|+1$, where $T$ is the set of tariff arcs.

\footnotetext{
* Dept of Quantitative Economics, Maastricht University, P.O.Box 616, 6200 MD Maastricht, The Netherlands. E-mail: s.vanhoesel@ke.unimaas.nl

$\dagger$ Dept of Quantitative Economics, Maastricht University, P.O.Box 616, 6200 MD Maastricht, The Netherlands. E-mail: a.vanderkraaij@ke.unimaas.nl

¥Università di Roma, La Sapienza, Via Buonarotti 12, 00185 Roma, Italy. E-mail: mannino@dis.uniroma1.it

$\S$ Departimento di Informatica Sistemi e Produzione, Università di Tor Vergata, Via del Politecnico 1, 00133 Roma, Italy. E-mail: oriolo@disp.uniroma2.it

IFrance Télécom R\&D, 39-40 rue du Général Leclerc, F-92131 Issy-Les-Moulineaux, France. E-mail: mustapha.bouhtou@rd.francetelecom.com
} 
This paper focuses on polynomially solvable special cases of the tarification problem. Some cases (all with linear pricing) have been described in earlier work. Labbé et al. [4] identified the case with one commodity where the path in the network taken by the client in the optimal solution is known a priori (fixed path linear tarification problem). Furthermore they show that for multiple commodities and one tariff arc (single arc linear tarification problem) the problem is also polynomially solvable. We generalize the latter case in three different ways.

For a linear pricing strategy, if the number of tariff arcs is upper bounded a priori, we show that the optimal solution can be obtained by solving a number (polynomially bounded by the number of commodities) of linear programs. We refer to this type of problem as the bounded arcs linear tarification problem. If we restrict the tariffs to be dependent on a single parameter (parametric tarification problem), we can solve the problem in polynomial time for an unlimited number of tariff arcs. Parametric tarification has an interesting special case, where all tariffs are restricted to be equal on all tariff arcs. Finally, the problem with multiple commodities and one tariff arc for the fixed charge pricing strategy (single arc fixed charge tarification problem) is shown to be polynomially solvable by a geometrical algorithm.

In the remainder, we first describe the general tarification problem we are dealing with and formulate it as a bilevel program. Subsequently, the notation used and the different pricing strategies studied are introduced. We briefly cover the properties of a remodeling of the network, introduced by Bouhtou et al. [1], and then illustrate each polynomially solvable special case.

\section{Model}

The underlying structure of the tarification problem is a directed graph $G=(N, A)$, where $A$ is partitioned into a set of tariff arcs $T$ and a set of fixed cost $\operatorname{arcs} F$. The clients form a set of commodities $K$, where each client $k \in K$ has a demand $d_{k}$ from node $s_{k}$ to node $t_{k}$. The tariff arcs belong to the leader and incur a toll for routing a client's demand. The fixed cost arcs are owned by other agents in the network, whose tariffs are demand dependent, but known a priori. We denote the cost of routing a demand $d$ on an arc $a \in F$ by $\gamma_{a}(d) \geq 0$. The pricing strategy on a tariff arc $a \in T$ is referred to by the nonnegative function $\rho_{a}(d)$, where $d$ is the demand to be routed on the tariff arc. We define for a commodity $k \in K$ the set of all possible paths from source to target by $P_{k}$. Denote by $T_{p}$, respectively $F_{p}$, the set of tariff arcs, respectively the set of fixed cost arcs, on a path $p \in P_{k}$. The revenue associated with a path $p$ for a demand $d$ is defined by

$$
\pi_{p}(d):=\sum_{a \in T_{p}} \rho_{a}(d)
$$

whereas the fixed cost associated with the path $p$ is given by:

$$
c_{p}(d):=\sum_{a \in F_{p}} \gamma_{a}(d)
$$

The tariffs on the arcs of $T$ are determined such that the total revenue of the leader is maximized. The clients on the network route their demands from source to destination according to the shortest path with respect to total cost, where the total cost of a path is defined as the sum of all the tariffs and fixed costs on the arcs of the path. Define by $l_{p}(d)=c_{p}(d)+\pi_{p}(d)$, the length of a path $p$. Whenever the client has a choice among multiple shortest paths with the same total cost but with different revenues for the leader, we suppose the client takes the shortest path that is most profitable to the leader. We furthermore suppose that a path using only fixed cost arcs exists for every client $k \in K$, since the problem is otherwise unbounded.

The general multiple commodities tarification problem, which holds for all pricing strategies studied, can be defined by the following bilevel program: 


$$
\begin{array}{ll}
\max & \sum_{k \in K} \pi_{p_{k}^{*}}\left(d_{k}\right) \\
\text { s.t. } & p_{k}^{*}=\arg \min _{p \in P_{k}} l_{p}\left(d_{k}\right) \quad \forall k \in K
\end{array}
$$

For the bilevel program in (3), at the upper level the revenue of the leader is maximized, while at the lower level the clients take the shortest path, given the tariffs determined at the upper level.

For linear tarification, the cost of routing a demand $d$ on the tariff arc $a \in T$ is determined by a variable cost only. Define for each arc $a \in T$ the tariff $t_{a} \geq 0$. The pricing strategy $\rho_{a}(d)$ on a tariff arc $a \in T$ is defined as follows:

$$
\rho_{a}(d)=t_{a} d
$$

Linear tarification has been extensively studied in the field of transportation. See for example Labbé et al. [4] and Brotcorne et al. [2], who have studied the optimization of tolls on a transportation network.

When dealing with parameterized tarification, the tariffs are dependent on a single parameter $\tau \geq 0$, for all tariff arcs. The pricing strategy on an arc $a \in T$ is given by:

$$
\rho_{a}(d)=\left(\alpha_{a}+\beta_{a} \tau\right) d
$$

where $\alpha_{a}, \beta_{a} \geq 0$ are constants for all arcs $a \in T$.

In case of fixed charge tarification, clients pay a fixed cost to be able to route their demands on the network. Furthermore, for each unit of traffic, the client incurs a cost per unit of traffic. Define by $f_{a} \geq 0$ the fixed cost on the arc and by $v_{a} \geq 0$ the cost per unit of traffic. For fixed charge tarification, the cost of routing a demand $d$ on an $\operatorname{arc} a \in T$ with tariffs $f_{a}$ and $v_{a}$ is given by:

$$
\rho_{a}(d)=\left\{\begin{array}{lll}
0 & \text { if } & d=0 \\
f_{a}+v_{a} d & \text { if } & d>0
\end{array}\right.
$$

The demands are known for all clients a priori. Hence, the cost of routing a demand $d$ on an arc $a \in F$ can be computed a priori, for any pricing strategy of the other agents in the network. Note that both $l_{p}(d)$ and $\pi_{p}(d)$ are linear functions for all pricing strategies studied.

For each commodity in the network, we use a remodeling of the network, referred to as the shortest paths graph model (SPGM), defined by Bouhtou et al. [1].

The definition of the SPGM for a customer is as follows: consider the original graph $G=(N, A)$ with the tariff $\operatorname{arcs}$ in $T \subseteq A$. For a client $k \in K$ with demand $d_{k}$ from $s$ to $t$, we define the graph $G^{*}=\left(N^{*}, A^{*}\right)$ and the tariff $\operatorname{arcs} T^{*} \subseteq A^{*}$. In this graph, the tariff arcs are separate arcs. So, tariff arcs with a common vertex are separated. Next, we construct the following fixed cost arcs. Note that between two tariff arcs, a client takes the shortest path using only fixed cost arcs in $G$. These paths are represented by fixed cost arcs in $G^{*}$, connecting the head of one tariff arc with the tail of another. From the source $s$ we construct arcs to all the tail nodes of the tariff arcs, and from all the head nodes we construct an arc to the destination $t$, again only if paths exist using only fixed arcs in $G$. For each commodity, the path using only fixed cost arcs from $s$ to $t$ is represented by the arc $(s, t)$. Note that we have supposed that this arc is always present, since the problem is otherwise unbounded. Any fixed cost arc in $A^{*}$ has a cost equal to the length of the shortest path between its end vertices in $G$, using only fixed cost arcs in $G$.

As shown by Bouhtou et al. [1], both models are equivalent. Concisely, for any path in the network $G$, there exists a path in the SPGM that is at least as good. Conversely, if a path exists 


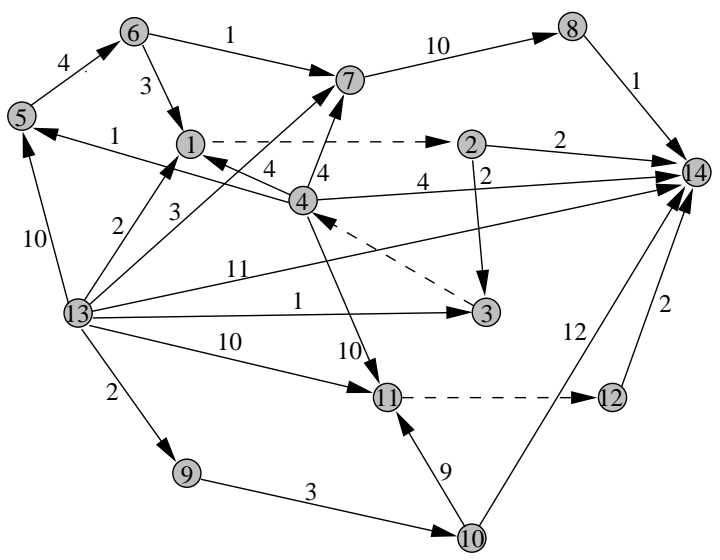

(a) Original network.

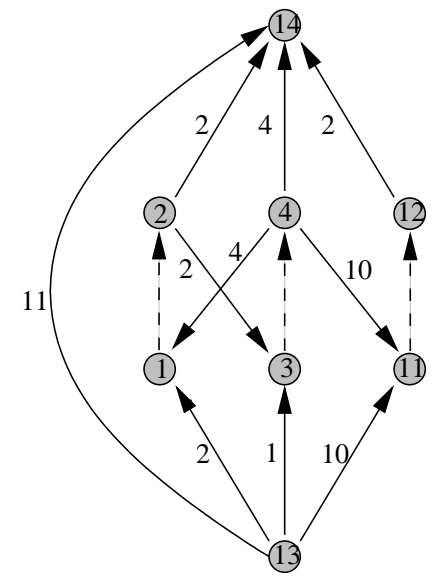

(b) SPGM.

Figure 1: The original network $G$ and the shortest paths graph model $G^{*}$.

in the SPGM, then a path with identical costs exists in the network $G$. Hence, an optimal solution in the SPGM has the same value in the original network. See for example the network depicted in figure 1(a). For this network, we are dealing with three tariff arcs, represented by the dashed arcs. The fixed cost arcs are represented by the solid arcs. The SPGM for a client with a demand from node 13 to node 14 is given in figure 1(b). If a path does not exist between two nodes in the original network, the corresponding fixed cost arc has infinite cost. In figure 1(b), we have chosen not to draw these infinite cost arcs. Note that the size of the network is $\mathcal{O}\left(|T|^{2}\right)$ for each commodity.

Since the SPGM only remodels the network by replacing the fixed cost arcs of the network, the SPGM holds not only for a linear pricing strategy, but for all pricing strategies on the tariff arcs where the cost of routing a client's demand on a fixed cost arc can be calculated a priori. For example, in case of a fixed charge pricing strategy, as long as the demand is known a priori, the cost of routing a client on a fixed cost arc can be calculated, enabling the calculation of all necessary shortest paths. The tariff arcs are just copied to the SPGM, including their pricing strategy.

\section{Bounded Arcs Linear Tarification Problem}

In this section, we study the complexity of the linear tarification problem with a bounded number of tariff arcs. The bilevel program defined in (3) is shown to be equivalent to a class of linear programs. Each linear program is constructed in such a way that the path taken by each client in the solution is fixed. Together, the linear programs in the class are equivalent to the linear tarification problem. The number of linear programs to solve is bounded by $|K|^{f(|T|)} g(|T|)$, while each linear program is of size $h(|T|)$. Note that the functions $f(|T|), g(|T|)$ and $h(|T|)$ depend only on the size of the set of tariff arcs. The number of linear programs to solve is therefore polynomially bounded by the number of commodities, whenever $|T|$ is upper bounded a priori. Since we can solve a linear program in polynomial time, the bounded arcs linear tarification problem is polynomially solvable.

For any client $k \in K$, consider two paths $p_{1}, p_{2} \in P_{k}$. If $p_{1}$ is to be the shortest of the two paths, the constraint $l_{p_{1}}\left(d_{k}\right) \leq l_{p_{2}}\left(d_{k}\right)$ must hold. Thus:

$$
c_{p_{1}}\left(d_{k}\right)+\pi_{p_{1}}\left(d_{k}\right) \leq c_{p_{2}}\left(d_{k}\right)+\pi_{p_{2}}\left(d_{k}\right) \Longleftrightarrow
$$




$$
\pi_{p_{1}}\left(d_{k}\right)-\pi_{p_{2}}\left(d_{k}\right) \leq c_{p_{2}}\left(d_{k}\right)-c_{p_{1}}\left(d_{k}\right)
$$

The constraint $l_{p_{1}}\left(d_{k}\right) \leq l_{p_{2}}\left(d_{k}\right)$ is of the form:

$$
\sum_{a \in T_{1}} t_{a}-\sum_{a \in T_{2}} t_{a} \leq b^{k}\left(p_{1}, p_{2}\right)
$$

Here, $b^{k}\left(p_{1}, p_{2}\right)$ is a constant and $T_{1}$ and $T_{2}$ are disjoint subsets of $T$. Note that $T_{1}$ contains the tariff arcs in $p_{1}$ not in $p_{2}$, and $T_{2}$ contains the tariff arcs in $p_{2}$ not in $p_{1}$. Tariff arcs on both paths do not appear in this constraint and hence we may assume $T_{1} \cap T_{2}=\emptyset$. The constant $b^{k}\left(p_{1}, p_{2}\right)$ is referred to in the remainder as the switching value for the pair $\left(p_{1}, p_{2}\right)$ for a client $k \in K$, since $p_{1}$ is shorter than $p_{2}$ if equation (8) holds and $p_{2}$ is shorter than $p_{1}$ if equation (8) does not hold. If $p_{1}$ and $p_{2}$ have the same set of tariff arcs, then $T_{1}=T_{2}=\emptyset$. In that case, the left hand side of (8) evaluates to zero. Thus, the sign of $b^{k}\left(p_{1}, p_{2}\right)$ determines which of the two paths is shortest, for all possible tariff values. We compute for each client $k \in K$, for all $p_{1}, p_{2} \in P_{k}$, the switching values $b^{k}\left(p_{1}, p_{2}\right)$.

We denote the set of all subsets of $T$ by $\mathcal{T}$. The set of all pairs of mutually disjoint sets of tariff arcs is then defined as:

$$
\mathcal{T}^{2}:=\left\{\left(T_{1}, T_{2}\right) \in \mathcal{T} \times \mathcal{T}: T_{1} \cap T_{2}=\emptyset\right\}
$$

Let $B$ be the increasingly ordered sequence of switching values $\left\{b^{k}\left(p_{1}, p_{2}\right) \mid p_{1} \in P_{k}, p_{2} \in P_{k}, k \in\right.$ $K\} \cup\{-\infty,+\infty\}$, say $B=\left(b_{0}, b_{1}, \ldots, b_{R}\right)$. For each pair $\left(T_{1}, T_{2}\right) \in \mathcal{T}^{2}$, we introduce an index in the ordered sequence $B$, say $r \in\{1, \ldots, R\}$.

For any $\left(T_{1}, T_{2}\right) \in \mathcal{T}^{2}$, we select an index, say $r\left(T_{1}, T_{2}\right) \in\{1, \ldots, R\}$. We consider the following set of constraints:

$$
b_{r\left(T_{1}, T_{2}\right)-1} \leq \sum_{a \in T_{1}} t_{a}-\sum_{a \in T_{2}} t_{a} \leq b_{r\left(T_{1}, T_{2}\right)} \quad \forall\left(T_{1}, T_{2}\right) \in \mathcal{T}^{2}
$$

Lemma 1. Given a choice $r\left(T_{1}, T_{2}\right) \in\{1, \ldots, R\}$ for all $\left(T_{1}, T_{2}\right) \in \mathcal{T}^{2}$, then for each client $k \in K$ there exists a path $p_{k}^{*} \in P_{k}$ that is the shortest path for all tariffs satisfying the constraints in (10), if (10) is not empty.

Proof. We prove that for each client $k \in K$ and each pair of paths $p_{1} \in P_{k}$ and $p_{2} \in P_{k}$, one of the two is shortest for any tariffs satisfying (10). For two paths with the same set of tariff arcs this is trivial, as noted previously, so we can restrict ourselves to paths for which this is not the case.

For any client $k \in K$ take two arbitrary paths $p_{1} \in P_{k}$ and $p_{2} \in P_{k}$. As we have seen previously, the constraint $l_{p_{1}}\left(d_{k}\right) \leq l_{p_{2}}\left(d_{k}\right)$ is equivalent to the constraint:

$$
\sum_{a \in T_{1}} t_{a}-\sum_{a \in T_{2}} t_{a} \leq b^{k}\left(p_{1}, p_{2}\right)
$$

where $b^{k}\left(p_{1}, p_{2}\right)$ is one of the switching values in the sequence $B$. Denote the index of this switching value by $\bar{r}$. For the constraints in (10), the pair $\left(T_{1}, T_{2}\right)$ in (11) has index $r\left(T_{1}, T_{2}\right)$.

If $\bar{r} \geq r\left(T_{1}, T_{2}\right)$, then

$$
b_{r\left(T_{1}, T_{2}\right)-1} \leq \sum_{a \in T_{1}} t_{a}-\sum_{a \in T_{2}} t_{a} \leq b_{r\left(T_{1}, T_{2}\right)} \leq b_{\bar{r}}
$$


implies that $l_{p_{1}}\left(d_{k}\right) \leq l_{p_{2}}\left(d_{k}\right)$. Conversely, if $\bar{r}<r\left(T_{1}, T_{2}\right)$, then $b_{\bar{r}} \leq b_{r\left(T_{1}, T_{2}\right)-1}$. Thus,

$$
b_{\bar{r}} \leq b_{r\left(T_{1}, T_{2}\right)-1} \leq \sum_{a \in T_{1}} t_{a}-\sum_{a \in T_{2}} t_{a} \leq b_{r\left(T_{1}, T_{2}\right)}
$$

implies that $l_{p_{1}}\left(d_{k}\right) \geq l_{p_{2}}\left(d_{k}\right)$. Concluding, for all tariffs satisfying the constraints in (10), there exists therefore a complete ordering of paths according to path length per commodity. For all $k \in K$, there is thus a path $p_{k}^{*}$ that is the shortest for all paths in $P_{k}$. The tariff arcs on this path yield a revenue of $\pi_{p_{k}^{*}}\left(d_{k}\right)$.

Now, consider (10) for a choice $r\left(T_{1}, T_{2}\right) \in\{1, \ldots, R\}$ for all $\left(T_{1}, T_{2}\right) \in \mathcal{T}^{2}$ and let the path $p_{k}^{*}$ be the shortest for all paths in $P_{k}$ for each client $k \in K$. The revenue associated with the path $p_{k}^{*}$ is given by the linear function:

$$
\pi_{p_{k}^{*}}\left(d_{k}\right)=\sum_{a \in T \cap p_{k}^{*}} t_{a} d_{k}
$$

For a choice $r\left(T_{1}, T_{2}\right) \in\{1, \ldots, R\}$ for all $\left(T_{1}, T_{2}\right) \in \mathcal{T}^{2}$, define the following linear program, referred to as $L P(r)$ :

$$
z_{L P(r)}= \begin{cases}\max _{t_{a}: a \in T} & \sum_{k \in K} \sum_{a \in T \cap p_{k}^{*}} t_{a} d_{k} \\ \text { s.t. } & b_{r\left(T_{1}, T_{2}\right)-1} \leq \sum_{a \in T_{1}} t_{a}-\sum_{a \in T_{2}} t_{a} \leq b_{r\left(T_{1}, T_{2}\right)} \quad \forall\left(T_{1}, T_{2}\right) \in \mathcal{T}^{2}\end{cases}
$$

Refer to the optimal value of the multiple commodities linear tarification problem for the bilevel program defined in (3) as $z_{M C T P}$.

\section{Theorem 1.}

$$
\max _{r\left(T_{1}, T_{2}\right)} z_{L P(r)}=z_{M C T P}
$$

Proof. Given $B$ and a choice of $r\left(T_{1}, T_{2}\right)$ for all $\left(T_{1}, T_{2}\right) \in \mathcal{T}^{2}$, an optimal solution (if it exists) to $L P(r)$ generates a feasible solution to $z_{M C T P}$. Therefore:

$$
z_{L P(r)} \leq z_{M C T P}
$$

Consider the optimal solution of $M C T P$. This yields a set of tariffs where for all clients $k \in K$ a path $p_{k}^{*} \in P_{k}$ is the shortest path. Given the optimal set of tariffs $t^{*}$, we define $\alpha_{\left(T_{1}, T_{2}\right)}$ :

$$
\sum_{a \in T_{1}} t_{a}^{*}-\sum_{a \in T_{2}} t_{a}^{*}=\alpha_{\left(T_{1}, T_{2}\right)} \quad \forall\left(T_{1}, T_{2}\right) \in \mathcal{T}^{2}
$$

Now, for all $\left(T_{1}, T_{2}\right) \in \mathcal{T}^{2}$, choose $\bar{r}_{\left(T_{1}, T_{2}\right)}$ such that:

$$
b_{\bar{r}_{\left(T_{1}, T_{2}\right)}-1} \leq \alpha_{\left(T_{1}, T_{2}\right)} \leq b_{\bar{r}_{\left(T_{1}, T_{2}\right)}} \quad \forall\left(T_{1}, T_{2}\right) \in \mathcal{T}^{2}
$$

Such an $\bar{r}_{\left(T_{1}, T_{2}\right)}$ exists for all $\left(T_{1}, T_{2}\right) \in \mathcal{T}^{2}$ and all clients $k \in K$ since the sequence $B$ has values ranging from $\langle-\infty,+\infty\rangle$. Given this choice of $\bar{r}_{\left(T_{1}, T_{2}\right)}$ for all $\left(T_{1}, T_{2}\right) \in \mathcal{T}^{2}$, the solution $t^{*}$ is feasible in $L P(\bar{r})$. Thus,

$$
z_{M C T P} \leq z_{L P(\bar{r})}
$$

From equations (17) and (20), it follows that

$$
z_{L P(\bar{r})}=z_{M C T P}
$$


We have shown that the optimal solution to the bilevel formulation of the linear tarification problem can be obtained by solving a number of linear problems as in (15). In the remainder of this section, we will compute bounds on the number of linear programs to be solved and on the size of each linear program.

Lemma 2. For each client $k \in K$, the number of paths in the network is bounded by e|T|!.

Proof. Given the shortest path graph model, the maximum number of paths for a client $k \in K$ is defined by selecting an ordered set (possibly empty) of tariff arcs. A set of $t$ arcs can be ordered in $t$ ! ways and be chosen in $\left(\begin{array}{c}|T| \\ t\end{array}\right)$ ways. Therefore, a bound on the number of paths is given by:

$$
\sum_{t=0}^{|T|}\left(\begin{array}{c}
|T| \\
t
\end{array}\right) t !=\sum_{t=0}^{|T|} \frac{|T| !}{(|T|-t) ! t !} t !=|T| ! \sum_{t=0}^{|T|} \frac{1}{(|T|-t) !}=|T| ! \sum_{t=0}^{|T|} \frac{1}{(t) !} \leq e|T| !
$$

Lemma 3. The number of elements in the sequence $B$ is bounded by $|K|(e|T| !)^{2}$.

Proof. Each element of the sequence $B$ is determined from a constraint of the form (8), where for a client $k \in K$ two paths $p_{1} \in P_{k}$ and $p_{2} \in P_{k}$ are compared. For a client $k \in K$, the number of comparisons is at most $\left|P_{k}\right|^{2}$. From lemma 2 we know that $\left|P_{k}\right| \leq e|T|$ !. Hence, the number of elements in the sequence $B$ is at most $|K|(e|T| !)^{2}$.

Theorem 2. Define $f(|T|)=4^{|T|}, g(|T|)=(e|T| !)^{8^{|T|}}$ and $h(|T|)=|T| 4^{|T|}$. The tarification problem with a linear pricing strategy can be solved by $|K|^{f(|T|)} g(|T|) h(|T|)$ linear programs, each of which is of size at most $h(|T|)$.

Proof. It follows from theorem 1 that we need to solve a number of linear programs as defined in (15). Each of the constraints in the linear program $L P(r)$, described in (15), is defined by a choice of $T_{1} \in T$ and $T_{2} \in T$, such that $T_{1} \cap T_{2}=\emptyset$. The number of possible subsets of $\left(T_{1}, T_{2}\right)$ is bounded by $2^{|T|} 2^{|T|}=4^{|T|}$, hence the number of constraints for each $L P(r)$ is bounded by $4^{|T|}$. For each constraint there is a choice of at most $|K|(e|T| !)^{2}$ right hand sides. Hence, the number of all possible linear programs $L P(r)$ is at most $\left.\left\{|K|(e|T| !)^{2}\right\}^{4^{|T|}}=|K|^{f(|T|)} g(|T|)\right)$, where $f(|T|)=4^{|T|}$ and $g(|T|)=(e|T| !)^{8^{|T|}}$. The size of each linear program $L P(r)$ is defined by the number of variables (at most $|T|$ ) and the number of constraints (at most $4^{|T|}$ ). Thus, the size of each linear program is bounded by $h(|T|)=|T| 4^{|T|}$.

\section{Parametric Tarification}

Another polynomially solvable variant of the tarification problem is the parametric tarification problem (PTP). For this problem we restrict the tariffs on all tariff arcs to a single parameter $\tau$ : $\rho_{a}(d)=\left(\alpha_{a}+\beta_{a} \tau\right) d$ for all $a \in T$.

Given a value for the parameter $\tau$, we can calculate the shortest path tree for each client. Knowing the shortest path taken by each client for this value of $\tau$, we can determine the revenue to the leader. To keep track of the changes of the shortest path for each client, we maintain a shortest path tree.

Finding the breakpoints in the shortest path tree for each client $k \in K$ corresponds to solving $|K|$ instances of the parametric shortest path problem, one for each commodity. Young and Faster [6] and Karp and Orlin [3] studied this problem and showed that the number of breakpoints is $\mathcal{O}\left(n^{2}\right)$. They furthermore described an algorithm which finds all these breakpoints in $\mathcal{O}(n m+$ $\left.n^{2} \log m\right)$. Here, $|V|=n$ and $|E|=m$. With this result, we can find all breakpoints in $\mathcal{O}(|K|(n m+$ $\left.n^{2} \log m\right)$ ). Denote these breakpoints by $\tau_{1}, \ldots, \tau_{B}$ and by $\pi_{k}\left(\tau_{i}\right)$ the profit to the leader from client 


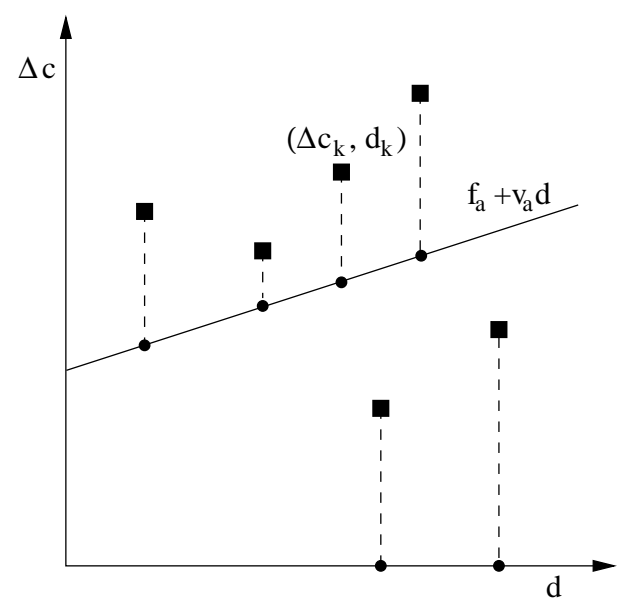

Figure 2: A Single Arc Fixed Charge Tarification.

$k \in K$ when the value of the parameter $\tau$ is set to $\tau_{i}, i \in\{1, \ldots, B\}$. If we denote the shortest path taken by the client $k \in K$ at parameter value $\tau_{i}$ by $p_{k}^{*}, \pi_{k}\left(\tau_{i}\right)$ is given by the following:

$$
\pi_{k}\left(\tau_{i}\right)=\sum_{a \in T \cap p_{k}^{*}}\left(\alpha_{a}+\beta_{a} \tau_{i}\right) d_{k}
$$

Now, let

$$
i^{*} \in \arg \max _{i}\left\{\sum_{k \in K} \pi_{k}\left(\tau_{i}\right): i \in 1, \ldots, B\right\}
$$

The optimal pricing strategy on each tariff $\operatorname{arc} a \in T$ is given by $\rho_{a}(d)=\left(\alpha_{a}+\beta_{a} \tau_{i^{*}}\right) d$. As (24) shows, the profit function of the leader is not necessarily continuous. Note furthermore that an interesting application of parametric tarification occurs when $\rho_{a}(d)=\tau d$ for all $a \in T$. For this particular problem, the tariffs are restricted to be equal on all tariff arcs.

\section{Single Arc Fixed Charge Tarification}

Although fixed charge tarification (see (6)) is more complex than linear tarification, we can solve the fixed charge tarification problem when dealing with a single tariff arc and multiple clients in polynomial time, using a geometrical algorithm.

For each commodity, we consider two paths. Let $p_{k}^{u}$ denote the shortest path taken by client $k \in K$ from node $s_{k}$ to node $t_{k}$, using only fixed arcs, whose cost $c_{p_{k}^{u}}\left(d_{k}\right)$ is an upper bound on the cost of any path from $s_{k}$ to $t_{k}$. Furthermore, let $p_{k}^{l}$ be the shortest path taken by client $k$ with smallest fixed cost from node $s_{k}$ to node $t_{k}$, taken by the client when the tariffs are equal to zero. Clearly, $c_{p_{k}^{l}}\left(d_{k}\right)$ is a lower bound on the cost of any path from $s_{k}$ to $t_{k}$. An upper bound on the revenue induced by each client $k \in K$ is therefore $c_{p_{k}^{u}}\left(d_{k}\right)-c_{p_{k}^{l}}\left(d_{k}\right)$. Denote this upper bound by $\Delta c_{k}=c_{p_{k}^{u}}\left(d_{k}\right)-c_{p_{k}^{l}}\left(d_{k}\right)$. Note that we can discard clients for which $\Delta c_{k}$ is equal to zero.

Consider figure 2 . In this figure, we are dealing with six commodities. All commodities $k \in K$ are plotted as the (square) points defined by $\left(d_{k}, \Delta c_{k}\right)$. The line in the figure represents a fixed charge tariff function $\rho_{a}(d)=f_{a}+v_{a} d$. A commodity $k \in K$ yields revenue to the leader if and only if $f_{a}+v_{a} d_{k} \leq \Delta c_{k}$. As we can see in the figure, four commodities (which are above the line) yield revenue to the leader, while two commodities do not. The revenue to the leader from each commodity is given by the dots in the figure. Refer to the set of points $\left(d_{k}, \Delta c_{k}\right)$ for all $k \in K$ as the set $U$. As the following lemma shows, to find the optimal fixed charge tariff function, we can restrict ourselves to consider only those tariff functions that contain at least two points in $U$. 
Lemma 4. Given is a set of clients $K$ and a single tariff arc with a fixed charge pricing strategy. The optimal fixed charge tariff function contains at least two points in $U$.

Proof. Without loss of generality, we assume that the optimal fixed charge tariff function, referred to as the function $\rho_{a}^{*}(d)=f^{*}+v^{*} d$, serves the clients in the set $S \subseteq K$. Thus, for the single tariff arc $a \in T, f^{*}+v^{*} d_{k} \leq \Delta c_{k}$ for all clients $k \in S$. Suppose that the tariff function $\rho_{a}^{*}(d)$ contains none of the points in $U$. In that case, we can increase $f^{*}$, yielding a higher profit. Now, suppose the function $\rho_{a}^{*}(d)$ contains exactly one point in $U$, say $\left(d_{k}^{*}, \Delta c_{k}^{*}\right)$. We will show that in that case, there exists a tariff function which contains at least two points in $U$, yielding at least as much revenue to the leader.

The tariff function $\rho_{a}^{*}(d)$ containing $\left(d_{k}^{*}, \Delta c_{k}^{*}\right)$ is given by the line $\rho_{a}^{*}(d)-\Delta c_{k}^{*}=v^{*}\left(d-d_{k}^{*}\right)$. The total revenue to the leader is thus:

$$
\sum_{k \in S}\left[\Delta c_{k}^{*}+v^{*}\left(d_{k}-d_{k}^{*}\right)\right]
$$

If we rotate the tariff function $\rho_{a}^{*}(d)$ around $\left(d_{k}^{*}, \Delta c_{k}^{*}\right)$ by increasing $v^{*}$ by $\Delta v$, then the difference in revenue is given by $\sum_{k \in S} \Delta v\left(d_{k}-d_{k}^{*}\right)$. Now, if $\sum_{k \in S}\left(d_{k}-d_{k}^{*}\right) \geq 0$, resp. $\sum_{k \in S}\left(d_{k}-d_{k}^{*}\right)<0$, then we can increase, resp. decrease, $v^{*}$ until the tariff function contains at least two points in $U$, yielding at least as much revenue to the leader.

Theorem 3. Given is a set of clients $K$ and a single tariff arc with a fixed charge pricing strategy. The optimal fixed charge tariff function can be found in $\mathcal{O}\left(n^{3}\right)$.

Proof. Given is the set of all clients $K$, where $|K|=n$, and the set of points $U$. From lemma 4 we know that the optimal fixed charge tariff function goes through at least two points in the set of points $U$. Since $|U|=n$, we therefore need to consider exactly $\left(\begin{array}{l}n \\ 2\end{array}\right)=n(n-1) / 2$ tariff functions. For each of these fixed charge tariff functions, we can calculate the revenue of the leader in $\mathcal{O}(n)$. Evaluating the revenue for the leader at all these possible lines allows us to find the optimal solution to our problem in $\mathcal{O}\left(n^{3}\right)$.

\section{Conclusion}

In this paper we introduced several new polynomially solvable instances of the general tarification problem as defined by Labbé et al. [4]. The first special case proposed is the multiple commodities with linear pricing strategies tarification problem, where the number of tariff arcs is bounded a priori (bounded arcs linear tarification problem). Second, we introduced the parametric tarification problem, which has a practical special case: for all tariff arcs, all tariffs are restricted to be equal in the optimal solution. Finally, we showed that the single arc fixed charge tarification problem is also a polynomially solvable special case.

Further research could extend the class of polynomially solvable special cases even further. One special case which could be interesting and extends the research presented in this paper is to bound the number of commodities a priori. Furthermore, the case with a fixed charge tarification pricing strategy and multiple tariff arcs, where the number of tariff arcs is upper bounded a priori remains open.

Besides giving more insight into the problem at hand, these polynomially solvable special cases lend themselves naturally to approximation algorithms. One further avenue of research is certainly to investigate the use of the algorithms devised here as approximation algorithms for more general problems. The approximation algorithm of Roch et al. [5] uses the fixed path linear tarification problem defined in Labbé et al. [4] to achieve a $\frac{1}{2} \log |T|+1$ performance guarantee for the single commodity linear tarification problem. It would be interesting to see if this bound can be improved for a constant factor, or whether there exists a (fully) polynomial time approximation scheme. 


\section{Acknowledgment}

This research was supported by France Télécom Research \& Development. The authors would also like to thank DONET for additional financial support.

\section{References}

[1] M. Bouhtou, S. van Hoesel, A. F. van der Kraaij, and J.L. Lutton. Tariff optimization in networks. Submitted to Journal on Computing, Meteor Research Memorandum, RM03011, 2003.

[2] L. Brotcorne, M. Labbé, P. Marcotte, and G. Savard. A bilevel model and solution algorithm for a freight tariff-setting problem. Transportation Science, 34(3):289 - 302, 2000.

[3] R.M. Karp and J. B. Orlin. Parametric shortest path algorithms with an application to cyclic staffing. Discrete Applied Mathematics, 3:37-45, 1981.

[4] M. Labbé, P. Marcotte, and G. Savard. A bilevel model of taxation and its application to optimal highway pricing. Management Science, 44:1608-1622, 1998.

[5] S. Roch, G. Savard, and P. Marcotte. Design and Analysis of an approximation algorithm for Stackelberg network pricing. Optimization Online, 2003.

[6] N. E. Young, R. E. Tarjan, and J. B. Orlin. Faster parametric shortest path and minimumbalance algorithms. Networks, 21:205-221, 1991. 